\title{
UN APPAREIL STANDARD POUR L'INSÉMINATION ARTIFICIELLE DES REINES D'ABEILLES 1
}

\section{STANDARDAPPARAT ZUR INSTRUMENTELLEN BESAMUNG DER BIENENKÖNIGIN 1}

\author{
F. RUTTNER, H. SCHNEIDER * et J. FRESNAYE ** \\ Institut für Bienenkunde, 637 Oberursel/Ts, Im Rothkopf 5 \\ * Außenstelle für Bienenzucht 357 Kirchhain, Evlenstr. 9 \\ ** Station expérimentale d'apiculture, \\ Centre de recherches d'Avignon, I.N.R.A., \\ 84140 Montfavet
}

\section{SUMMARY \\ STANDARD INSEMINATION APPARATUS FOR QUEEN HONEY-BEES}

Based on the insemination apparatus designed by MACKensen and RoBerts, a standardised model was developed allowing a quick and easy insemination of queens. This equipment has been tested by several institutes and insemination stations since 1969 . The modifications concern the queen holder, the fixation of hooks and syringe and the mechanical movement of the syringe.

\section{TEXTE FRANÇAIS}

\section{RÉSUMÉ}

A partir de l'appareil pour l'insémination artificielle des reines mis au point par MACKENSEN et RoBERTs, un modèle standard a été développé lequel permet un travail rapide et commode. Cet équipement a été testé par plusieurs instituts de recherches apicoles et par des stations de fécondation depuis 1969. Les modifications concernent le dispositif de contention de la reine, la fixation des crochets et de la seringue ainsi que les mouvements mécaniques de celle-ci.

\section{I. - INTRODUCTION}

L'insémination artificielle des reines est devenue l'auxiliaire indispensable de tout programme de recherches génétiques ou de sélection consacré à l'abeille.

1. Dies ist eine deutsch-französische Gemeinschaftsarbeit. Wegen ihrer Bedeutung für die Weiterentwicklung der Besamungstechnik wird der volle Text sowohl in französischer wie in deutscher Sprache gebracht.

1. Le présent travail est le fruit d'une coopération franco-allemande. En raison de son importance pour le développement futur de l'insémination artificielle des reines d'abeilles il est présenté dans son intégralité en français et en allemand. 
Réservée pendant de nombreuses années aux travaux de laboratoire, elle entre progressivement dans le domaine pratique. Des stages de formation d'inséminateurs ont lieu dans différents pays. Des apiculteurs souhaitent être en mesure de féconder des reines par des mâles connus, soit dans le but de sélection ou d'hybridation soit pour éviter l'achat de reines à l'étranger. En même temps, un plus grand nombre de laboratoires s'intéressent à cette technique qui permet d'apporter une plus grande rigueur dans les travaux.

Face à cette situation nouvelle d'une vulgarisation plus importante de l'insémination artificielle, il nous paraît utile de proposer un nouvel appareil qui réunisse les éléments les plus intéressants disséminés sur divers modèles préexistants et bénéficie de l'expérience d'une équipe de spécialistes ayant de nombreuses années de pratique et plusieurs milliers d'inséminations à leur actif. Conçu à partir du modèle de base de MAckensen et RoBerts il réunit toute une série de perfectionnements issus des recherches de différents laboratoires (Fig. 1).

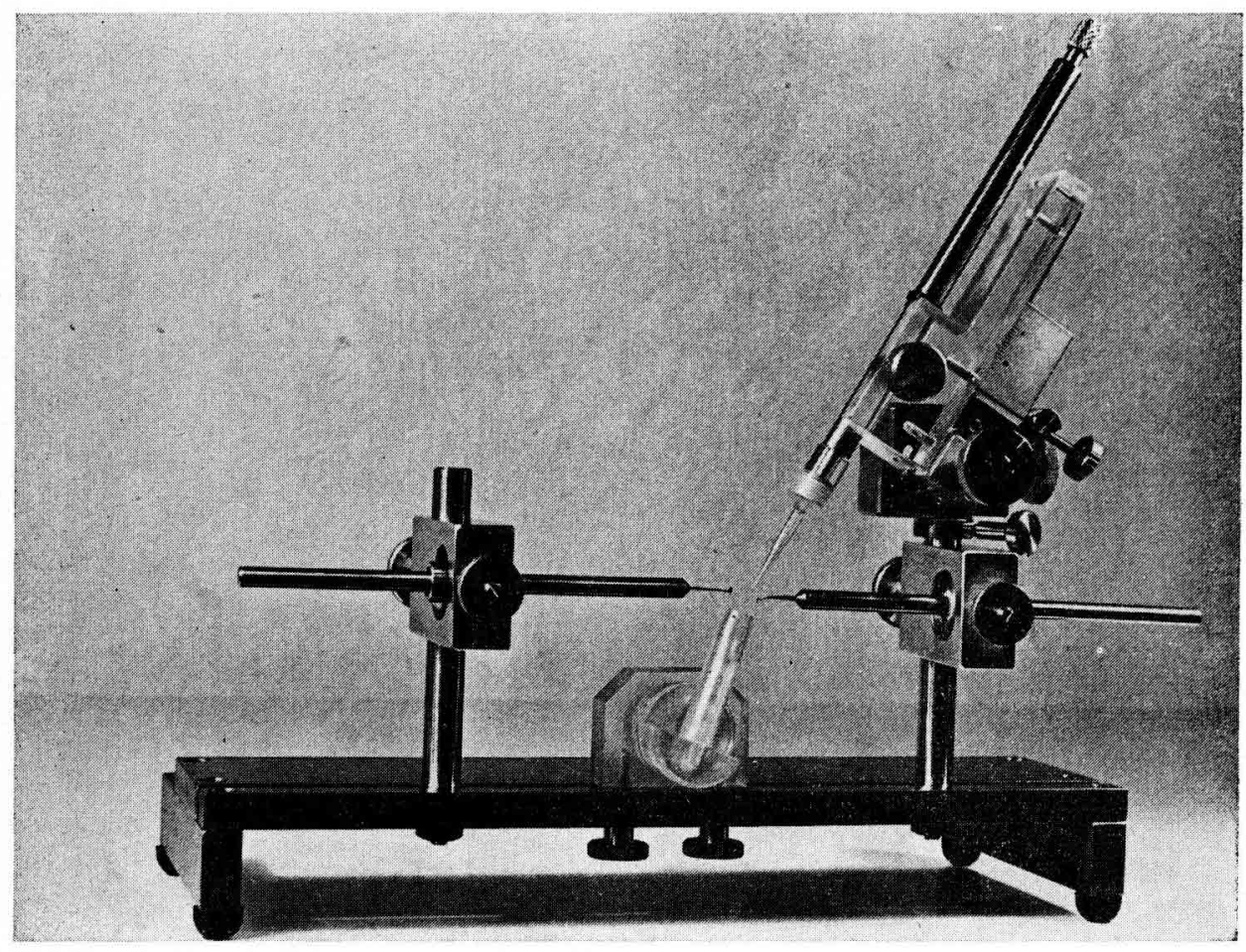

Авв. 1. - Besamungsapparat Standardmodell

Fig. 1. - Appareil standard pour l'insémination des reines d'abeilles 
Les principaux critères qui ont présidé à l'élaboration de l'appareil sont les suivants :

10 Il doit être très pratique et utilisable aussi bien par les spécialistes chevronnés que par des personnes d'une dextérité moyenne et peu expérimentées.

$2^{o}$ Il doit être aussi simple que possible, sans toutefois nuire à son efficacité, afin que sa construction en série soit peu onéreuse. Pour la même raison on utilise, dans toute la mesure du possible, des éléments standardisés se trouvant dans le commerce.

$3^{\circ}$ Il doit permettre une grande rapidité des différentes opérations afin de réduire le temps nécessaire aux inséminations et de diminuer les frais de production des reines ainsi fécondées.

On peut considérer que l'insémination artificielle instrumentale a débuté avec les travaux de WATson en 1927. Depuis, différents types d'appareils ont été proposés aux utilisateurs. Certains sont très simples, donc généralement peu coûteux, mais exigent une grande dextérité et une longue pratique. D'autres sont très élaborés, nécessitent un appareillage complexe et coûteux; ils ne requièrent théoriquement qu'une adresse manuelle plus courante.

A la suite des travaux de Watson, puis de Nolan (1937), Mackensen et RoBerts (1948) construisirent un appareil de conception simple, très répandu actuellement. Puis, de nouveaux modèles plus ou moins apparentés dans leur conception aux précédents apparurent, notamment ceux deVESELY (1961) de Velthuis (1970), de Weber. L'appareil de Laidlaw (1949-1953) est différent, tous les mouvements étant commandés par des micromanipulateurs.

\section{II. - PRINCIPAUX ÉlÉmENTS DE L'APPAREIL STANDARD}

\section{1. - Le dispositif de contention de la reine}

Dans les appareils de Mackensen et Roberts ainsi que de Vesely, le dispositif de contention de la reine est composé d'un tube de plexiglas et d'un piston auquel le tuyau d'arrivée du $\mathrm{CO}^{2}$ est adapté. Le tube est introduit par dessous dans le bloc de fixation. Rutrner (1968) a proposé un dispositif pour fixer la reine plus commodément. Il offre l'avantage d'éviter le déplacement du tuyau d'arrivée du $\mathrm{CO}^{2}$; en outre, il permet de faire pivoter à tout moment la reine sur son axe longitudinal sans avoir à desserrer de vis. Simplifié depuis sa description originale ce dispositif (Fig. 2) est fixé sur le socle de l'appareil au moyen de la plaque de base $P$. Au centre de celle-ci se trouve un orifice qui est en rapport avec la conduite de gaz et, par devant, un disque 


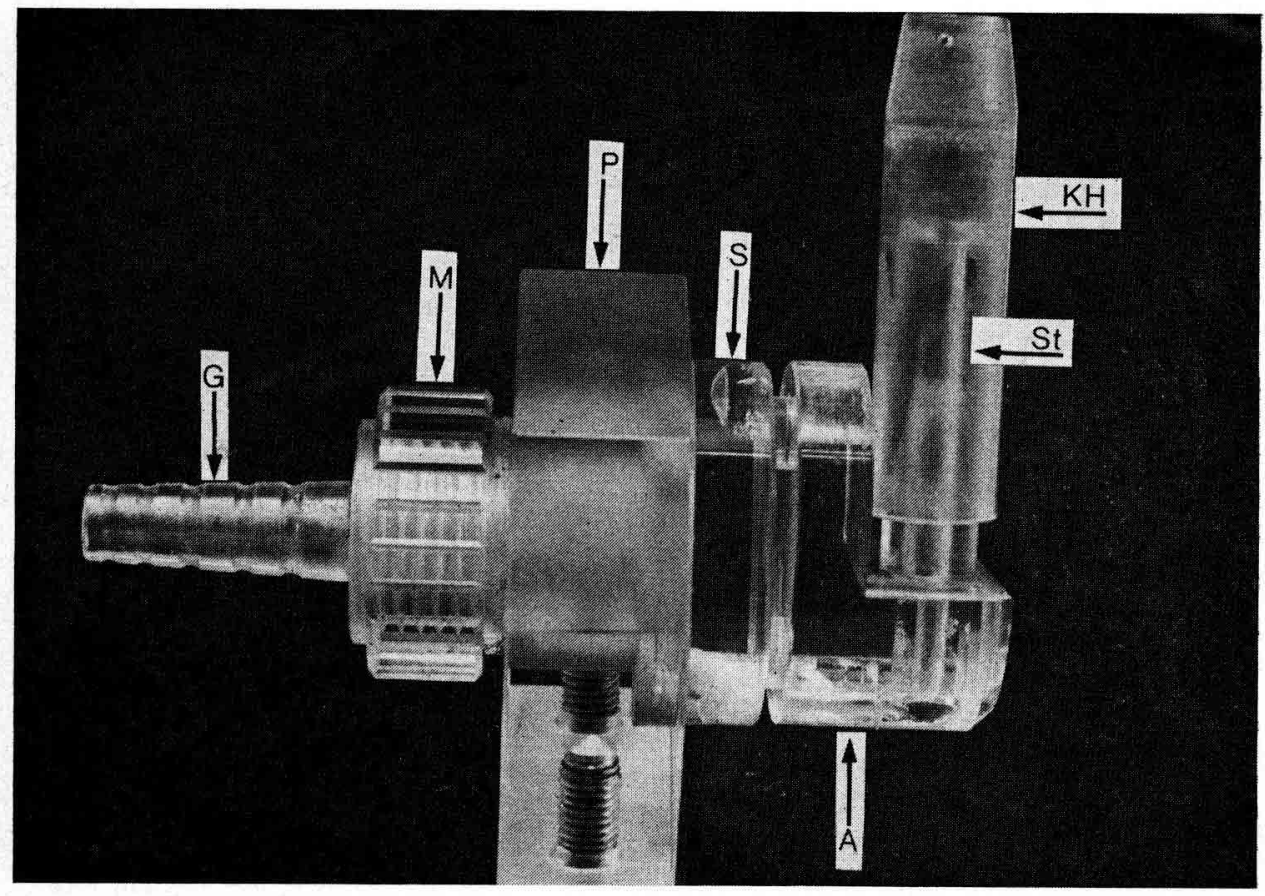

AвB. 2. - Narkosekammer (Plexiglas)
A Aufsteckplatte
G Gaszuleitung
KH Königinhalter
M Mutter
P Grundplatte
S Scheibe am Gaszuleitungsrohr
St Stopper

Fig. 2. - Chambre d'anesthésie (en plexiglas)
A. Plaque de fixation
G. Arrivée du gaz
KH. Appareil de contention de la reine
M. Écrou
P. Plaque basale
S. Disque vers l'arrivée du gaz
St. Arrêt 
(S) pour la fixation du dispositif de contention de la reine. La liaison entre le disque et la plaque de fixation se fait par tenons et trous comme dans le système “ Lego »; elle est donc facile à établir. L'inclinaison correcte de l'axe du dispositif de contention de la reine est fixée au moyen de l'écrou $M$.

\section{2. - L'articulation des crochets, ventral et dorsal}

Dans l'appareil de Laiduaw, les crochets sont commandés par des glissières à crémaillères formant micromanipulateurs. Dans ceux de MACKensen et de VESELY, les mouvements sont directement commandés à la main, simplement secondée par deux axes pivotant, l'un verticalement, l'autre horizontalement. Au cours de travaux d'insémination, puis de stages de formation d'inséminateurs, il nous est apparu que la plupart des élèves s'accommodaient plus rapidement des mouvements directs que de ceux qui sont transmis par les micromanipulateurs. La difficulté de transformation du mouvement circulaire en mouvement rectiligne en est en partie la cause. En outre, les mouvements que l'on peut exécuter, bien que plus délicats à assurer, sont beaucoup plus variés dans le système manuel direct. Enfin, le prix de revient des appareils à commande directe est beaucoup moins élevé que celui des appareils à micromanipulateurs.

Un dispositif à rotule, (Fig. 3) proposé par SCHneider, permet d'améliorer la souplesse et la continuité des mouvements directs qui sont ainsi apparentés aux articulations de la main. Le jeu des manches des crochets résultant de la compression des ressorts qui se produit dans l'appareil de Mackensen et Roberts est ainsi évité. Les crochets sont ceux proposés par Mackensen; utilisés sur tous les appareils, leur forme est imposée par la morphologie des reines.

\section{3. - La seringue}

La seringue mise au point par Mackensen est idéale dans sa simplicité et elle est aujourd'hui utilisée universellement sans modifications. La pointe en plexiglas a une contenance d'environ 10 $\mu$ l. Elle n'est pas vissée directement dans le tube métallique de la seringue mais par l'intermédiaire d'un manchon de plexiglas. Pour pouvoir utiliser les pointes qui, généralement, proviennent des U.S.A. on a conservé le pas américain. L'ouverture de $0,75 \mathrm{~mm}$ se rétrécit vers l'extrémité jusqu'à 0,15 à $0,17 \mathrm{~mm}$. Le diamètre externe ne doit pas dépasser à l'extrémité $0,28 \mathrm{~mm}$. Une description de la fabrication des pointes d'après Mackensen se trouve dans Ruttner, 1969.

Les pointes en verre ont l'inconvénient d'être plus fragiles mais l'avantage d'être plus faciles à fabriquer et à nettoyer. Leur parois étant plus fines elles ont un diamètre externe plus faible pour une même lumière. Les tubes capillaires ou les micro-pipettes calibrées sont étirés dans la flamme et on les coupe 


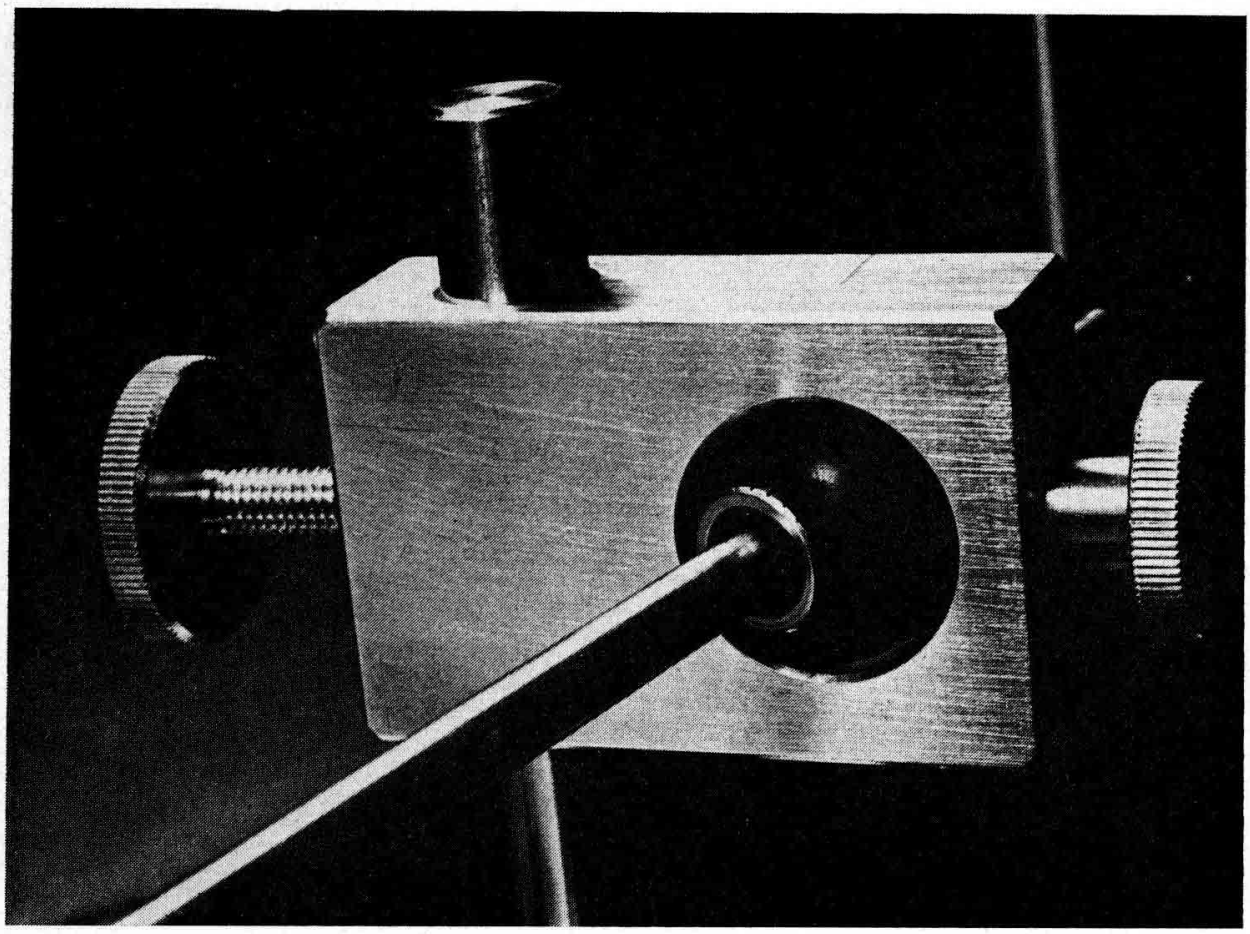

Авв. 3. - Häkchenhalter mit Kugelgelenk.

Eine verstellbare konische Tefloneinlage in der Kugel (vgl. Abb. 4., T) gewährleistet eine weich Friktion für den Häkchengriff.

FIG. 3. - Support des crochets avec rotule

Un cône mobile en téflon placé dans la rotule permet une friction douce du manche du crochet (Voir Fig. 4 T)

au diamètre désiré. On élimine les éclats de verre dans la flamme ou avec un abrasif. Le capillaire de verre est enfoncé à la base dans un petit tube de matière plastique et vissé dans le manchon au moyen d'un socle en plexiglas.

\section{4. - Descente de la seringue}

La descente de la seringue dans la chambre vaginale de la reine doit être précise. Ce mouvement extrêmement délicat justifie l'utilisation d'une commande à crémaillère. Le fait que la fixation de la seringue n'a pas besoin d'être desserrée au cours de la descente représente un gain de temps. Nous utilisons des crémaillères de platine de microscope.

\section{5. - Inclinaison de la seringue}

Des mouvements de faible amplitude peuvent être nécessaires avant ou après la descente de la seringue dans la chambre vaginale. Il faut alors prendre 
de grandes précautions pour ne pas blesser la reine. Le dispositif commandé par vis, proposé par VESELY, autorise des mouvements très précis à l'intérieur de la chambre vaginale (Fig. 4, St). L'utilisation d'une sonde vaginale est rendue inutile.

\section{6. - Fixation de la seringue}

La seringue est fixée dans une sorte d'étau $(R)$ par une vis. Elle s'enlève latéralement, ce qui réduit beaucoup les risques de détérioration de la pointe lors des manipulations.

La possibilité d'enlever facilement la seringue de son support permet d'envisager différents modes d'utilisation de l'appareil. Si l'utilisateur travaille seul il doit exécuter lui-même toutes les opérations. Il prélève les mâles dans leur ruche (ceux-ci peuvent d'ailleurs avoir été encagés plusieurs jours à l'avance) puis les reines dans les ruchettes ou nuclei. Ensuite il procède au prélèvement du sperme et à l'insémination. Il peut également préparer plusieurs doses de sperme dans autant de seringues avant de prélever plusieurs reines à la fois et de pratiquer l'insémination de celles-ci. On perd un certain temps à passer de l'une à l'autre de ces diverses activités; en contrepartie le travail est moins contraignant et permet une certaine détente du fait de la diversité des occupations. Une équipe de deux personnes permet de séparer les activités apicoles de l'insémination proprement dite. Une équipe de trois personnes permet un véritable travail à la chaîne avec une augmentation du rendement non négligeable. Un seul spécialiste est indispensable pour l'insémination des reines; un débutant peut assurer le prélèvement du sperme dans plusieurs seringues; un apiculteur assure l'approvisionnement en reines et en mâles ainsi que le retour des reines dans leur colonie. L'utilisation de deux ou de plusieurs seringues permet de varier les combinaisons et les possibilités de travail avec dans tous les cas une plus grande souplesse de celui-ci et un rendement accru tout en ne nécessitant qu'un seul appareil d'insémination.

Les croquis techniques détaillés permettant la construction de l'appareil se trouvent dans la seconde édition de l'ouvrage « Die instrumentelle Besamung der Bienenkönigin ” par F. Ruttner, éditions Apimondia, Bucarest.

L'appareil peut être obtenu à l'adresse suivante :

Aussenstelle für Bienenzucht

D 357 KIRGHHAIN

Erlenstrasse 9

R.F.A.

Reçu pour publication en juin 1974. 


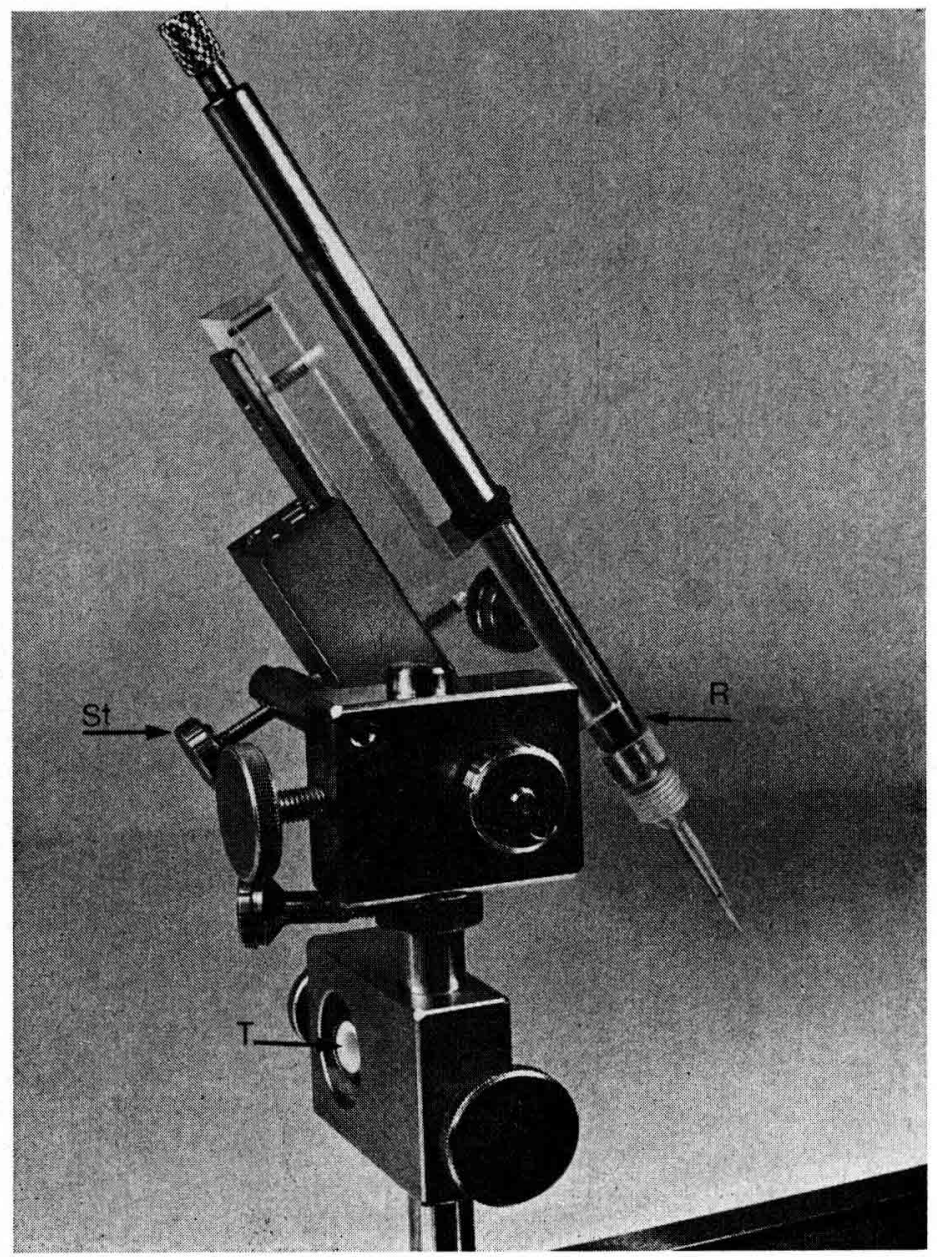

Aв8. 4. - Spritzenblock

$\mathbf{R}$ Rinne aus Plexiglas zur Befestigung der Spritze

St Stellschraube zur Veränderung des Neigungswinkels

$\mathbf{T}$ Tefloneinlage in der Führung für den Häkchengriff.
Fig. 4. - Bloc seringue

$R$ Gouttière en plexiglas pour la fixation de la seringue

St Vis pour le règlage de l'inclinaison de la seringue

T Revêtement de téflon dans le conduit du manche du crochet

\section{Deutscher Text}

\section{I. - EINLEITUNG}

Die instrumentelle Besamung der Königinnen ist zu einer unentbehrlichen Arbeitsmethode bei allen genetischen Untersuchungen oder Selektionsprogrammen bei der Biene geworden. Während sie viele Jahre hindurch fast 
ausschliesslich in Laboratorien angewandt wurde, findet sie jetzt in zunehmenden Masse Eingang in die Praxis. In verschiedenen Ländern werden Kurse zur Ausbildung von Besamungstechnikern abgehalten, es wurden staatliche oder private Besamungsstationen gegründet. Die Bienenzüchter bemühen sich im Rahmen einer planmässigen Selektion oder Hybridisierung um kontrollierte Paarungen, oder sie wollen auf diese Weise den Zukauf von Königinnen von auswärts vermeiden. Gleichzeitig beschäftigt sich eine zunehmende Zahl von Laboratorien mit dieser Technik, die eine grössere Genauigkeit bei den Arbeiten ermöglichen.

Angesichts der zunehmenden Verbreitung der instrumentellen Besamung erscheint es uns angebracht, das verbesserte Modell eines Besamungsapparates vorzustellen, das sich seit einigen Jahren bei mehreren Arbeitsgruppen ausgezeichnet bewährt. Er hat sich auch bei uns in jahrelanger praktischer Erfahrung, gewonnen im Verlaufe von mehreren tausend Besamungen, als das bisher bequemste und einfachste Modell erwiesen. Ausgehend von dem Grundtyp von Mackensen und Roberts vereinigt es eine Reihe von Verbesserungen in sich, die in verschiedenen Laboratorien entstanden sind.

Bei der Modifizierung des Apparates liessen wir uns von folgenden Gesichtspunkten leiten :

1. Der Apparat muss in der Hand des routinierten Spezialisten wie in der eines weniger geübten Laien von nur mittelmässiger Geschicklichkeit in gleicher Weise geeignet sein.

2. Für eine Serien-Herstellung zu möglichst geringen Kosten muss er so einfach als möglich konstruiert sein, ohne dass jedoch die Leistungsfähigkeit darunter leidet. Aus demselben Grunde werden soweit als möglich standardisierte, im Handel erhältliche Bestandteile verwendet.

3. Der Apparat soll eine rasche Durchführung der einzelnen Handgriffe ermöglichen, um Zeitaufwand und Kosten für eine Besamung zu senken.

Die instrumentelle Besamung der Bienenkönigin geht auf die Arbeiten von Watson im Jahre 1927 zurück. Seither wurden verschiedene Typen von Apparaten angeboten. Einige sind sehr einfach und infolge dessen wenig kostspielig, aber sie verlangen grössere Geschicklichkeit und lange Erfahrung. Andere sind sehr ausgeklügelt, mit einer komplizierten und kostspieligen Konstruktion, die aber trotzdem eine betsächtliche manuelle Geschicklichkeit voraussetzen.

Aufbauend auf den Arbeiten von Watson und später von Nolan (1937) haben Mackensen und Roberts 1948 einen Apparat einfacher Bauart konstruiert, der weite Verbreitung gefunden hat. Später wurden verschiedene andere Modelle veröffentlicht, die sich mehr oder weniger stark an die ursprüngliche Konzeption anlehnten, so die Apparate von Vesely (1961) und von 
Velthuis (1970). Nur der Apparat von Laidlaw (1949-1953) ist verschieden, bei ihm werden alle Bewegungen mit Hilfe von Feintrieben durchgeführt.

\section{H. - DIE WICHTIGSTEN KONSTRUKTIONSELEMENTE DES STANDARDAPPARATES}

\section{1. - Narkosekammer}

Bei den Apparaten von Mackensen und Roberts und von Vesely besteht die Narkosekammer aus einem Röhrchen aus Plexiglas und einem "Stopper », an dem der Zuleitungsschlauch für das $\mathrm{CO}_{2}$-Gas befestigt ist. Das Röhrchen wird von unten in den Fixierungsblock eingeführt. RutrNer hat 1968 ein bequemer zu handhabendes Modell vorgeschlagen. Bei diesem bleibt die Gaszuführung beim Wechsel der Königin unberührt, ausserdem kann die Königin zu jeder Zeit um ihre Längsachse gedreht werden, ohne dass eine Fixierungsschraube gelockert werden müsste. Die Narkosekammer (Abb. 2), die seit ihrer ersten Beschreibung noch vereinfacht wurde, ist mittels der Grundplatte $\mathbf{P}$ am Stativ des Apparates befestigt.

Durch eine Bohrung in ihre Mitte führt das Gaszuleitungsrohr, das vorne eine Scheibe (S) zur Befestigung des Königinhalters trägt. Die leicht lösbare Verbindung zwischen Scheibe und Aufsteckplatte erfolgt durch zwei Nippel und Bohrungen, wie bei dem Baukastensystem “ Lego ». Die richtige Neigung der Achse des Königinhalters wird durch Anziehen der Mutter M fixiert.

\section{2. - Bewegliche Befestigung der beiden Häkchen}

Bei dem Apparat von LaIDlaw werden die Häkchen durch Zahntrieb einzeln wie bei einem Mikromanipulator bewegt, bei den Apparaten von Mackensen und Vesely werden die Bewegungen direkt von Hand dirigiert, wobei Drehungen um zwei Achsen (vertikal und horizontal) möglich sind. Bei einem Vergleich der beiden Systeme machten wir vor allem bei der Ausbildung von Besamungstechnikern die Erfahrung, dass die direkte manuelle Führung der Häkchen rascher gelernt wird als die Übertragung durch den Mikromanipulator.

Ausserdem ist der Bewegungsspielraum bei direkter manueller Betätigung wesentliche vielfältiger. Ein weiterer Nachteil des als Mikromanipulator gebauten Apparates ist sein wesentlich höherer Preis.

Die von Schneider entwickelte Befestigung der Häkchen durch ein Kugelgelenk ermöglicht, ähnlich wie bei den Gelenken der menschlichen Hand, sehr freie und weichkontinuierliche Bewegungen. Das “ Spiel 》 der Häkchengriffe, wie es beim Typ Mackensen und Roberts infolge Kompression der Feder auftritt, ist hier vermieden. Die Häkchen, deren Form durch die Mor- 
phologie der Königin bestimmt wird, sind wie bei sämtlichen anderen Apparaten dieselben wie bei Mackensen und Roberts.

\section{3. - Die Spritze}

Die von Mackensen entwickelte Spritze ist in ihrer effektiven Einfachheit ideal für die Besamung und wird heute ganz allgemein ohne Veränderung verwendet. Die Spritzenspitze aus Akrylglas fasst etwa $10 \mu \mathrm{l}$. Sie wird nicht direkt in das Metallrohr der Spritze geschraubt, sondern mittels einer Verbindungsmuffe ebenfalls aus Akrylglas. Um die meist aus den U.S.A. bezogenen Spitzen benutzen zu können, wurde hier das Zollgewinde beibehalten. Die Bohrung von $0,75 \mathrm{~mm}$ verjüngt sich an der Spitze auf 0,15-0,17 mm. Der äussere Durchmesser soll an der Spitze $0,28 \mathrm{~mm}$ nicht überschreiten. Eine Beschreibung der Herstellung der Spitzen gibt Mackensen in Ruttner 1969.

Spitzen aus Glas haben den Nachteil, dass sie leichter brechen, aber die Vorteile, dass sie leichter herzustellen und zu reinigen sind, und dass sie wegen der geringeren Wandstärke bei gleichem Lumen einen geringeren Aussendurchmesser haben. Glaskapillaren oder kalibrierte Mikropipetten werden über der Flamme ausgezogen und an der Stelle mit dem gewünschten Durchmesser abgebrochen. Die Beseitigung der Bruchkanten erfolgt durch vorsichitges Erhitzen oder durch Schleifen. Die Glaskapillare wird mit ihrer Basis in einen kurzen Abschnitt eines dünnen Kunststoffschlauches gesteckt und mittels eines Sockels aus Akrylglas in die Verbindungsmuffe geschraubt.

\section{4. - Das Einführen der Spitze in den medianen Ovidukt}

Das Einführen der Spitze in den medianen Ovidukt der Königin muss sehr exakt erfolgen. Deshalb ist zur Bedienung der Spritze die Verwendung eines Feintriebes gerechtfertigt. Dass die Fixierung der Spritze beim Einführen nicht gelockert werden muss, bedeutet eine Zeitersparnis.

Wir verwenden Feintriebe für Mikroskop-Kreuztische (z. B. von der Fa. Robra-Optik-Foto GmbH, 8 München, Postfach 720).

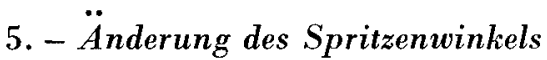

Vor oder nach dem Einführen der Spritze in die Vaginalkammer können Bewegungen kleiner Amplitude nötig sein. Der von VESELY vorgeschlagene Schraubmechanismus erlaubt sehr präzise Änderungen des Neigungswinkels der Spritze (Abb. 4, St). Die Verwendung einer Vaginalsonde wird dadurch unnötig. 


\section{6. - Befestigung der Spritze}

Die Spritze wird mittels einer Schraube in einer Rinne (R) fixiert, somit kann sie auch lateral entfernt werden. Das bedeutet einen Zeitgewinn und eine Verringerung des Risikos, beim Auswechseln der Spritze die empfindliche Spitze zu beschädigen.

Infolge dieser raschen und bequemen Auswechselbarkeit der Spritze können verschiedene Arbeitsmethoden mit diesem Apparat ins Auge gefasst werden. Arbeitet der Benutzer allein, so muss er alle Operationen selbst ausführen. Er entnimmt die Drohnen aus ihren Völkern (falls sie nicht schon mehrere Tage vorher gekäfigt worden sind). Dann wird der Samen entnommen und sie Insemination durchgeführt.

Man kann aber auch, um Zeit zu gewinnen, Spermadosen in mehreren Spritzen in einem Zug vorbereiten und anschliessend damit mehrere Königinnen hintereinander besamen. Jede dieser Methoden hat ihre Vor- und Nachteile. Beim ständigen Wechsel von einer Tätigkeit zur anderen verliert man zwar etwas Zeit. Andererseits aber ist diese Arbeitsweise weniger beanspruchend, da man sich beim Übergang von einer Tätigkeit zur anderen entspannen kann.

In einer Arbeitsgruppe von zwei Personen kann die Arbeit an den Bienen von der Insemination getrennt werden. Eine Gruppe von drei Personen ermöglicht ein richtiges Arbeiten am Fliessband und damit eine erhebliche Leistungssteigerung. Es genügt ein einziger Spezialist für die Besamung der Königinnen, ein Anfänger nimmt das Sperma in mehreren Spritzen auf und ein Imker bringt Königinnen und Drohnen, bzw. er setzt die besamten Königinnen in ihre Völker zurück. Bei Benutzung von zwei oder mehreren Spritzen können die Arbeitsmethoden in vielfältiger Weise modifiziert werden, mit dem Ergebnis einer grösseren Elastizität in Hinblick auf die Erfordernisse und einer grösseren Zahl besamter Königinnen, obwohl dazu nicht mehr als ein einziger Besamungsapparat erforderlich ist.

Die detaillierten technischen Zeichnungen zur Selbstherstellung des Apparates sind in der 2.Auflage von F. Ruttrer, « Die instrumentelle Besamung der Bienenkönigin », Apimondia Verlag Bukarest, publiziert.

Erhältlich ist der Apparat bei der Aussenstelle für Bienenzucht, 357 Kirchhain, Erlenstr. 9. 


\section{RÉFÉRENCES BIBLIOGRAPHIQUES}

LAIDLAW H.-H., 1949, Development of precision instruments for artificial insemination of queen-bees J. econ. Entomol. 42 (2) 254-261.

Laidlaw H.-H., 1949, New instruments for artificial insémination of queen-bees Amer. Bee $J$. 89 (12) 566-567.

LAIDLAW H.-H., 1953, An anesthetization chamber for the artificial insemination of queenbees J. econ. Entomol. 46 (1) 167.

Mackensen O., Roberts W.-C., 1948, A manual for the artificial insemination of queen-bees. U.S.D.A. Bur. Ent. and Plant. Quar. E.T. 250.33 p.

Mackensen O., 1948, A new syringe for the artificial insemination of queen-bees. Amer. Bee J. 88 (8) 412.

NoLAN W.-J., 1937, Improved apparatus for inseminating queen-bees by the WATson method J. econ. Entomol. 57 (4) 581-583.

Ruttner F., 1968, Insemination artificielle de la reine d'abeille. Ann. Abeille 11 (4) 241-320 (ainsi que Allemand, Anglais, Russe 1969 Apimondia. 80 pages.

VeLthuis H.-H.-W., et SommeiJer M.-J. 1970, Einige Modifikationen in der instrumentellen Besamung von Bienenköniginnen. Apidologie 1 (3) 343-346.

VESELY V., 1961, Towards the problem of artificial insemination of queen-bees. Zoologicke Lisby (10) 203-210.

Watson L.-R., 1927, Controlled mating in the honeybee Rep. Sta. Apiarist Iowa 36-41. 